\title{
Review article: effect of bile salt pool composition on hepatic and biliary functions
}

\author{
N. CARUlli, M. BERTOLOTti, F. CARUBBi, M. CONCARI, P. MARTELLA, L. CARUlli \& \\ P. LORIA
}

Department of Internal Medicine, University of Modena, Modena, Italy

\section{SUMMARY}

The enterohepatic recirculation of bile salts exerts important regulatory effects on many hepatic, biliary and intestinal functions; such regulation is likely to depend, to a large extent, on the physical-chemical property of hydrophobicity of the recirculating pool. The present review summarizes the main experimental evidence carried out by our research group over the past two decades, in the attempt to investigate systematically the relationships between structural properties and biological effects of bile acids in humans. Hydrophobic bile acids (chenodeoxycholic acid, deoxycholic acid), but not hydrophilic acids (ursodeoxycholic acid), significantly suppressed hepatic activity of HMG-CoA reductase, the limiting step of cholesterol synthesis, and in vivo cholesterol $7 \alpha$-hydroxylation, the limiting step of bile acid synthesis. The output of biliary cholesterol and phospholipid was also directly related to the hydrophobicity of the bile acid pool. Finally, treatment with chenodeoxycholic acid, but not with ursodeoxycholic acid, significantly decreased gallbladder emptying rates. When turning to the in vitro model of HepG2 cells, hydrophobic bile acids were found to induce greater cytotoxic and proapoptotic effects. From this series of studies, we conclude that the regulatory effects of bile acids on the liver and biliary tract are largely dependent on the hydrophobic-hydrophilic balance of the recirculating bile acid pool.

\section{INTRODUCTION}

Bile salts, due to their physical-chemical properties and the efficient mechanism for their transport across the intestine and the liver, are molecules well preserved within the anatomic compartment made by liver, biliary tract, intestine and portal circulation. This enterohepatic circulation (EHC) of bile salts is thus a highly economic circuit of molecules that contributes to the regulation of several hepatic, biliary and intestinal functions. ${ }^{1}$

In order to understand its functions, the EHC of bile salts has to be considered both quantitatively and qualitatively. In humans, the pool of bile salts varies from 2 to $4 \mathrm{~g}$ and recirculates in the EHC 6-

Correspondence to: Dr N. Carulli, Department of Internal Medicine, Via del Pozzo, 71, 41100 Modena, Italy.

E-mail: carulli@unimo.it
10 times a day. The result is that some $12-36 \mathrm{~g}$ of bile salts flow through the liver and intestine each day.

In addition, individual bile salt molecules have a different balance between their hydrophobic (steroid nucleus) and hydrophilic moieties ( $\mathrm{OH}$ substituents and side chain amino terminal), depending mainly on the number, position and orientation of the $\mathrm{OH}$ groups in the molecule and on the conjugation with either glycine or taurine. Ranking of common bile salts in order of hydrophobicity is: monohydroxy (lithocholic acid, LCA) $>$ dihydroxy (deoxycholic acid, DCA; chenodeoxycholic acid, CDCA) > trihydroxy acid (cholic, CA). Glycine conjugates are more hydrophobic than taurine conjugates.

Ursodeoxycholic acid (UDCA), a dihydroxy bile acid epimer of CDCA, is the most hydrophilic acid, 
owing to the presence of an $\mathrm{OH}$ group $(7 \beta$ instead of $7 \alpha$ position) located close to the hydrophobic site of the molecule.

The hydrophobic-hydrophilic balance ${ }^{2,3}$ determines many physical-chemical characteristics of bile acids, including critical micellar concentrations and detergent properties, and bears on the biological functions that bile salts contribute to the regulation of, recirculating in the EHC.

In humans, the bile salt pool is made, in physiological conditions, by CA and CDCA (in equal proportions, nearly $40 \%$ of the total), DCA (20\%) and traces of LCA and UDCA. Thus the hydrophobic-hydrophilic balance of the pool is shifted toward hydrophobicity.

In this short review we will consider some hepatic and biliary functions affected by the EHC of bile salts in humans, depending on the composition of the pool; emphasis will be given to the gall-bladder motor function.

\section{HEPATIC CHOLESTEROL METABOLISM}

The main contribution to body cholesterol comes from synthesis by the liver. The rate-limiting step in the synthetic process, the conversion of HMG-CoA to mevalonate, is catalysed by HMG-CoA reductase. Studies on human liver preparations have shown that HMG-CoA reductase is depressed in subjects treated short-term with hydrophobic bile acids, whereas hydrophilic ones such as UDCA seem to produce an increased enzyme activity, probably owing to a shift of the bile acid pool towards hydrophilicity. ${ }^{4,5}$

In humans, bile acids, end products of hepatic cholesterol degradation themselves, have been shown to regulate their own synthesis by a negative feedback mechanism. In fact subjects treated with cholestyramine, a bile acid sequestrant resin, show a dose-dependent increase of cholesterol $7 \alpha$ hydroxylation rate, the first committed and rate-limiting step in the pathway from cholesterol to bile acid. ${ }^{6,7}$

In contrast, subjects with cholestasis display a reduced rate of $7 \alpha$ hydroxylation of cholesterol that returns to normal values once cholestasis is relieved. ${ }^{8}$ In addition, studies in which subjects were treated with individual bile acids demonstrated that hydrophobic, but not hydrophilic, bile acids are able to suppress $7 \alpha-$ hydroxylation of cholesterol. ${ }^{6}$

\section{BILIARY LIPID SECRETION}

In humans, as in many animal species, biliary secretion of cholesterol and phospholipids is a function of bile acid secretion. ${ }^{9}$ The mechanism currently held responsible for this secretory coupling is that of solubilization of canalicular membrane microdomains due to bile acids present within the canalicular lumen. ${ }^{10}$ In this way cholesterol-phospholipid vesicles enter bile canaliculi where they undergo solubilization in mixed micelles.

Such a mechanism is supported by early observations by our group obtained in a systematic study carried out on T-tube subjects. ${ }^{11,12}$ In this model, with its inherent limitations, it was shown that changes in composition of the bile acid pool, brought about by acute duodenal infusion of individual bile acids, associated with variations of cholesterol and phospholipid secretion into the bile. Hydrophobic bile acids, such as DCA or CDCA, induced a lipid secretion significantly greater than that observed during secretion of hydrophilic bile acids such as CA or UDCA.

In the same study, evidence of the interaction between bile acids and the canalicular membrane was provided by the observation that secretion of a canalicular ecto enzyme, such as alkaline phosphatase, was related to biliary lipid output.

Subsequent studies, carried out on experimental animals, confirmed that the hydrophobic-hydrophilic balance of the bile acid pool is a main determinant of biliary lipid secretion. ${ }^{13}$

\section{REGULATION OF LIVER MASS}

Liver injury associated with cholestasis has been attributed to the retention of bile acids in the liver. Indeed, in an in vitro model using cell cultures hydrophobic bile acids have been shown to be cytotoxic. $^{14,15}$

Although cytotoxicity of bile acids may be related to membrane disruptive effects from their detergent properties, it seems that non-detergent mechanisms are also involved. ${ }^{16,17}$ In fact, our study using HepG2 cells and concentrations of hydrophobic bile acids far below those necessary to induce enzyme release has shown that hydrophobic bile acids depress $\left[{ }^{3} \mathrm{H}\right]$ thymidine incorporation, and increase DNA degradation and apoptosis. ${ }^{18}$

Indeed, whereas widespread liver cell necrosis is not a prominent feature in most cholestatic liver diseases, it 

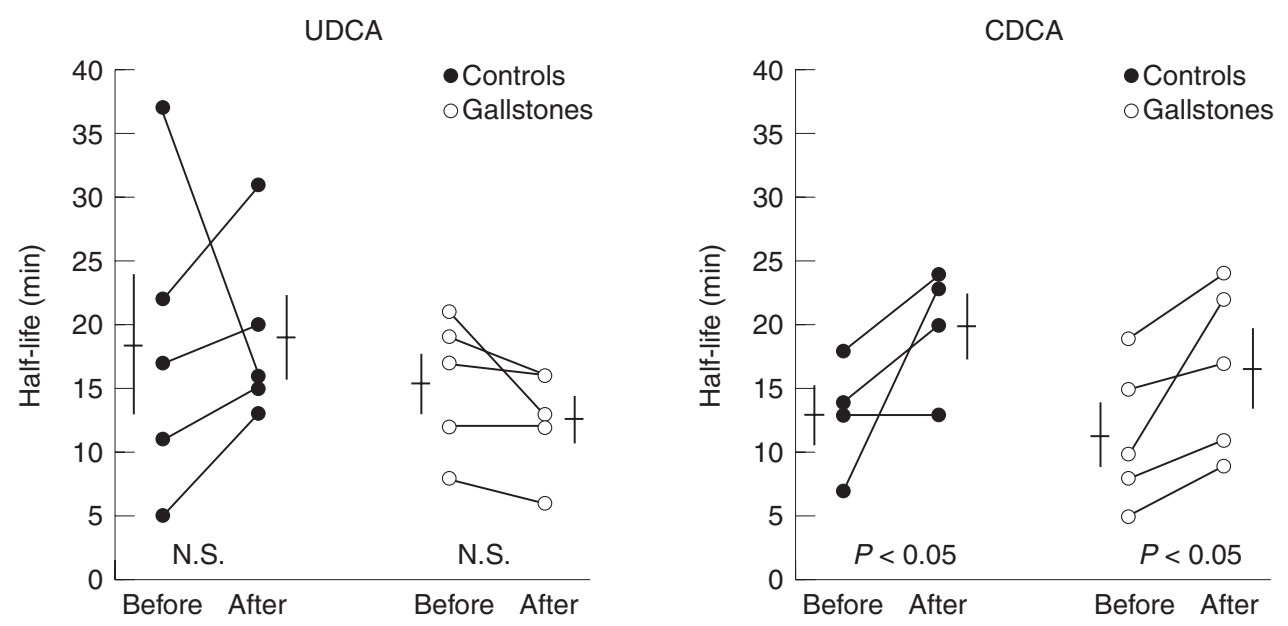

Figure 1. Effect of treatment with UDCA or CDCA on gall-bladder motor function in subjects with or without cholesterol gallstones. Bile acids $(10 \mathrm{mg} / \mathrm{kg})$ were administered for 4 weeks. Gall-bladder contraction was induced by a standard liquid meal. The half-life of gall-bladder emptying, reported on the ordinate, was evaluated by ultrasonography.

seems that apoptosis occurs more frequently than necrosis. ${ }^{19}$ It could be speculated that in physiological conditions hydrophobic bile acids could also contribute to the regulation of liver cell mass through induction of apoptosis.

\section{BILE ACID POOL COMPOSITION AND GALL- BLADDER MOTOR FUNCTION}

Gall-bladder hypomotility is currently held as a key determinant, in addition to cholesterol supersaturation and nucleation of cholesterol crystals, in the pathogenesis of cholesterol stones. ${ }^{20}$

Failure of the gall-bladder to fill and empty properly has been well established in animal models of cholelithiasis. ${ }^{21,22}$ Recently, altered gall-bladder motor function was also demonstrated clearly in gallstone patients by measuring gall-bladder bile turnover using a combination of cholescintigraphy and ultrasonography. ${ }^{23}$ The increased diversion of hepatic bile into the duodenum, due to the hypomotility of the gall-bladder, leads to an increased intestinal metabolism of bile acids, with the consequence that the bile acid pool enriches with secondary bile acids such as deoxycolate. This, in turn, could suppress bile acid synthesis and induce a greater biliary lipid secretion. ${ }^{6,11}$ However, in the sequence linking bile cholesterol supersaturation to gall-bladder hypomotility, it is matter of debate which of the two represents the primary event.
Alternatively, both of them might be contributed to by bile acid pool composition. In this line of reasoning a series of studies has examined the direct effect of individual bile acids on gall-bladder motility in different experimental settings. Thimister et al. ${ }^{24}$ reported that acute duodenal infusion of CDCA in healthy volunteers is able to inhibit CCK- or bombesin-stimulated gallbladder emptying. The effect could possibly be attributed to a diminished sensitivity of the target organ to CCK, caused by circulating hydrophobic CDCA.

In a similar study conducted by our group, ${ }^{25}$ subjects with cholesterol gallstones were treated for 4 weeks with CDCA or UDCA to obtain enrichment of the bile acid pool with the administered bile acid. CDCA-, but not UDCA-, treated subjects showed a decreased gallbladder emptying compared with pre-treatment values (Figure 1). The changes of gall-bladder motility induced by bile acids seem to be a direct effect related to the bile acid structure.

Using gall-bladder muscle strips of subjects with cholesterol stones, Stolk et al. ${ }^{26}$ demonstrated that the response of a muscle strip to either acetylcholine or CCK was impaired by addition of bile salts, either unconjugated or tauroconjugated; the suppression of contractility was related directly to the bile salt hydrophobicity, being none with UDCA and greatest with DCA.

In a similar in vitro study, using guinea-pig gallbladder smooth muscle, $\mathrm{Xu}$ etal. ${ }^{27}$ showed that contractility, stimulated either by CCK, bethanechol, $\mathrm{KCl}$ or electric field, was depressed by bile salts in a 
concentration-dependent manner and in order of increasing hydrophobicity of the bile salt used.

The mechanism by which bile salts induce changes of gall-bladder contractility is not defined. Acting at a micromolar range of concentration, either circulating or luminal bile salts may be responsible for the effect on the gall-bladder. The relevance of the reported findings on the pathophysiology of gall-bladder motor function in subjects with cholesterol stones is not straightforward.

In a study on gall-bladder motor function conducted on subjects with cholesterol gallstones, Stolk et al. ${ }^{28}$ were able, on the basis of post-prandial gall-bladder emptying evaluated by ultrasonography, to group subjects as strong or weak contractors, suggesting that hypomotility of the gall-bladder plays an initiating role, in the sequence of events leading to gallstone formation, only in some cases. However, the bile acid pool composition in the two groups of subjects did not show any significant difference.

In addition, Behar et al. ${ }^{29}$ reported that muscle strips obtained from human gall-bladder harbouring cholesterol stones show a reduced contractility compared with that observed in muscle strips from gall-bladder containing pigment stones, speculating that excess cholesterol in bile might diffuse in the gall-bladder wall and impair muscle contractility.

In conclusion, hypomotility of the gall-bladder plays a key role in the process of gallstone formation; the pathogenesis of impaired gall-bladder motor function is possibly multifactorial, including an increased hydrophobicity of bile acid pool composition.

\section{CONCLUSIONS}

The overall evidence from studies on experimental animals and humans supports the view that most of the physiological regulatory effects of $\mathrm{EHC}$ of bile salts are directly or indirectly related to the hydrophobichydrophilic balance of the bile salt pool.

\section{REFERENCES}

1 Vlahcevic ZR, Heuman DM, Hylemon PB. Physiology and pathophysiology of entrohepatic circulation of bile acids. In: Zakim D \& Boyer TD, eds. Hepatology: A Textbook of Liver Disease. Philadelphia: W.B. Saunders Company: 1996; 376417.

2 Armstrong MJ, Carey MC. The hydrophobic-hydrophilic balance of bile salts. Inverse correlation between reversephase high performance liquid chromatographic mobilities and micellar cholesterol-solubilizing-capacities. J Lipid Res 1982; 23: 70-80.

3 Heuman DM. Quantitative estimation of the hydrophilichydrophobic balance of mixed bile salt solutions. J Lipid Res 1989; 30: 719-30.

4 Carulli N, de Ponz Leon M, Zironi F, Iori R, Loria P. Bile acid feeding and hepatic sterol metabolism: effect of deoxycholic acid. Gastroenterology 1980; 79: 637-41.

5 Carulli N, de Ponz Leon M, Zironi F, et al. Hepatic cholesterol and bile acid metabolism in subjects with gallstones: comparative effects of short-term feeding of chenodeoxycholic and ursodeoxycholic acid. J Lipid Res 1980; 21: 35-43.

6 Bertolotti M, Abate N, Loria P, et al. Regulation of bile acid synthesis in humans: effect of treatment with bile acids, cholestyramine or simvastatin on cholesterol 7-hydroxylation rates in vivo. Hepatology 1991; 14: 830-7.

7 Bertolotti M, Abate N, Bertolotti S, et al. Effect of aging on cholesterol 7-hydroxylation in humans. J Lipid Res 1993; 34: 1001-7.

8 Bertolotti M, Concari M, Carulli L, et al. Obstructive cholestasis inhibits the rates of cholesterol 7-hydroxylation in humans in vivo. In: Paumgartner G, Stiehl A, Gerok W, eds. Bile Acids in Hepatobiliary Diseases: Basic Research and Clinical Application. Dordrecht: Kluwer Academic Publishers: 1997; 284-7.

9 Wagner CI, Trotman BW, Soloway RD. Kinetic analysis of biliary lipid excretion in man and dog. J Clin Invest 1976; 57 : 473-7.

10 Verkade HJ, Vonk RJ, Kuipers F. New insights into the mechanism of bile acid-induced biliary lipid secretion. Hepatology 1995; 21: 1174-89.

11 Carulli N, Loria P, Bertolotti M, et al. Effect of acute changes of bile acid pool composition on biliary lipid secretion. J Clin Invest 1984; 74: 614-24.

12 Loria P, Carulli N, Medici G, et al. Effect of ursocholic acid on bile lipid secretion and composition. Gastroenterology 1986; 90: 865-74.

13 Bilhartz LE, Dietschy JM. Bile salt hydrophobicity influences cholesterol recruitment from rat liver in vivo when cholesterol synthesis and lipoprotein uptake are constant. Gastroenterology 1988; 95: 771-9.

14 Scholmerich J, Becher MS, Schmidt K, et al. Influence of hydroxylation and conjugation of bile salts on their membrane-damaging properties: studies on isolated hepatocytes and membrane vesicles. Hepatology 1984; 4: 661-6.

15 Carubbi F, Guicciardi ME, Concari M, et al. Hepatotoxic and hepatoprotective effects of bile salts (BS) depend on their physico-chemical properties. Hepatology 1993; 18: 313 A.

16 Spivey JR, Bronk SF, Gores GJ. Glycochenodeoxycholateinduced lethal hepatocellular injury in rat hepatocytes. Role of ATP depletion and cytosolic free calcium. J Clin Invest 1993; 92: $17-24$.

17 Patel T, Bronk SF, Gores GJ. Increases of intracellular magnesium promote glycodeoxycholate-induced apoptosis in rat hepatocytes. J Clin Invest 1994; 94: 2183-92. 
18 Guicciardi ME, Grassilli E, Concari M, et al. Effect of bile salts in HepG2 cells on cell proliferation and induction of apoptosis. Hepatology 1996; 24: 367A.

19 Columbano A. Cell death: current difficulties in discriminating apoptosis from necrosis in the context of pathological processes in vivo. J Cell Biochem 1995; 58: 181-90.

20 Carey MC. Formation and growth of cholesterol gallstones: the new synthesis. In: Fromm H, Leuschner U, eds. Bile Acids Cholestasis Gallstones. Dordrecht: Kluwer Academic Publishers: 1996; 147-75.

21 Doty JE, Pitt HA, Kuchenbecker SL, DenBesten L. Impaired gallbladder emptying before gallstone formation in the prairie dog. Gastroenterology 1983; 85: 168-74.

22 Roslyn JJ, DenBesten L, Thompson JE, Cohen K. Chronic cholelithiasis and decreased bile salt pool size: cause or effect? Am J Surg 1980; 139: 119-24.

23 Carey MC, Robins SJ. Bile production and secretion. In: Stein JH, ed. Internal Medicine, 4th edn. St Louis: Mosby-Year Book Inc.: 1993; 532-9.

24 Thimister PWL, Hopman WPM, Tangerman A, Rosenbusch G, Willems HL, Jansen JBM. Effect of intraduodenal bile salt on pancreaticobiliary responses to bombesin and to cholecystokinin in humans. Hepatology 1998; 28: 1454-60.

25 Tripodi A, Loria P, Carulli N. Effect of chenodeoxycholic (CDCA) and ursodeoxycholic (UDCA) acid administration on gallbladder motility in man. Gastroenterology 1988; 94: A602 (abstract).

26 Stolk MFJ, Van de Heijning BJM, Van Erpecum KJ, Verheem A, Akkermans LMA, vanberge-Henegouwen GP. Effect of bile salts on in vitro gallbladder motility: preliminary study. Ital J Gastroenterol 1996; 28: 105-10.

27 Xu Q-W, Freedman SM, Shaffer EA. Inhibitory effect of bile salts on gallbladder smooth muscle contractility in the guinea pig in vitro. Gastroenterology 1997; 112: 1699-706.

28 Stolk MFJ, Van Erpecum KJ, Renooij W, Portincasa P, Van de Heuning BJM, vanBerge-Henegouwen GP. Gallbladder emptying in vivo, bile composition, and nucleation of cholesterol crystals in patients with cholesterol gallstones. Gastroenterology 1995; 108: 1882-8.

29 Behar J, Lee KY, Thompson WR, Biancani P. Gallbladder contraction in patients with pigment and cholesterol stones. Gastroenterology 1989; 97: 1479-84. 